\title{
Allelic dilution obscures detection of a biologically significant resistance mutation in EGFR-amplified lung cancer
}

\author{
Jeffrey A. Engelman, ${ }^{1,2,3}$ Toru Mukohara,4,5 Kreshnik Zejnullahu, ${ }^{4,5}$ Eugene Lifshits, ${ }^{6}$ \\ Ana M. Borrás, ${ }^{7}$ Christopher-Michael Gale, ${ }^{3}$ George N. Naumov, ${ }^{6}$ Beow Y. Yeap, ${ }^{1}$ \\ Emily Jarrell, ${ }^{3}$ Jason Sun,, 4,5 Sean Tracy, ${ }^{4,5}$ Xiaojun Zhao, ${ }^{5}$ John V. Heymach, ${ }^{8}$ \\ Bruce E. Johnson, ${ }^{4,5,9}$ Lewis C. Cantley,2,3 and Pasi A. Jänne ${ }^{4,5,9}$
}

\begin{abstract}
${ }^{1}$ Massachusetts General Hospital Cancer Center, Boston, Massachusetts, USA. 2Department of Systems Biology, Harvard Medical School, Boston, Massachusetts, USA. ${ }^{3}$ Department of Signal Transduction, Beth Israel Deaconess Medical Center, Boston, Massachusetts, USA. ${ }^{4}$ Lowe Center for Thoracic Oncology and ${ }^{5}$ Department of Medical Oncology, Dana-Farber Cancer Institute, Boston, Massachusetts, USA. ${ }^{6}$ Department of Surgery and Vascular Biology Program, Children's Hospital Boston, Boston, Massachusetts, USA. ${ }^{7}$ Translational Research Laboratory, Department of Medical Oncology, Dana-Farber Cancer Institute, Boston, Massachusetts, USA. ${ }^{8}$ Department of Thoracic/Head and Neck Oncology, University of Texas M. D. Anderson Cancer Center, Houston, Texas, USA. ${ }^{9}$ Departments of Medicine, Brigham and Women's Hospital and Harvard Medical School, Boston, Massachusetts, USA.
\end{abstract}

\begin{abstract}
EGFR is frequently mutated and amplified in lung adenocarcinomas sensitive to EGFR inhibitors gefitinib and erlotinib. A secondary mutation, T790M, has been associated with acquired resistance but has not been shown to be sufficient to render EGFR mutant/amplified lung cancers resistant to EGFR inhibitors. We created a model for studying acquired resistance to gefitinib by prolonged exposure of a gefitinib-sensitive lung carcinoma cell line (H3255; EGFR mutated and amplified) to gefitinib in vitro. The resulting resistant cell line acquired a T790M mutation in a small fraction of the amplified alleles that was undetected by direct sequencing and identified only by a highly sensitive HPLC-based technique. In gefitinib-sensitive lung cancer cells with EGFR mutations and amplifications, exogenous introduction of EGFR T790M effectively conferred resistance to gefitinib and continued ErbB-3/PI3K/Akt signaling when in cis to an activating mutation. Moreover, continued activation of PI3K signaling by the PIK3CA oncogenic mutant, $\mathrm{p} 110 \alpha \mathrm{E} 545 \mathrm{~K}$, was sufficient to abrogate gefitinib-induced apoptosis. These findings suggest that allelic dilution of biologically significant resistance mutations may go undetected by direct sequencing in cancers with amplified oncogenes and that restoration of PI3K activation via either a T790M mutation or other mechanisms can provide resistance to gefitinib.
\end{abstract}

\section{Introduction}

The EGFR is a member of a family of closely related growth factor receptor tyrosine kinases that includes EGFR (ErbB-1), HER2/neu (ErbB-2), HER3 (ErbB-3), and HER4 (ErbB-4). As EGFR is expressed in a majority of non-small cell lung carcinomas (NSCLCs), it has been an attractive target for the development of therapeutic agents (1-3). The small-molecule EGFR tyrosine kinase inhibitors (TKIs), including gefitinib (Iressa; AstraZeneca) and erlotinib (Tarceva; OSI Pharmaceuticals), have been evaluated in clinical trials for patients with NSCLC. Both agents cause partial responses in $10 \%-20 \%$ of all NSCLC patients (4-7). Tumors that possess activating mutations and/or amplification of the EGFR locus appear to be particularly sensitive to EGFR TKIs (8-14). In fact, lung cancers with EGFR mutations often harbor concurrent EGFR amplifications $(13,14)$. NSCLC cell lines in which EGFR is mutated and amplified, including $\mathrm{HCC} 827$ and $\mathrm{H} 3255$, are exquisitely sensitive in vitro to EGFR

Nonstandard abbreviations used: ca-Akt, constitutively active Akt; Del/T790M, EGFR with a T790M mutation cis to an exon 19 deletion mutation; DHPLC, denaturing HPLC; GR, gefitinib resistant; L858R/T790M, EGFR with a T790M mutation cis to an L858R mutation; MTS, 3-(4,5-dimethylthiazol-2-yl)-5-(3-carboxymethoxyphenyl)-2-(4-sulfophenyl)-2H-tetrazolium; NSCLC, non-small cell lung carcinoma (cancer); p-, phosphorylated; PARP, poly ADP-ribose polymerase; PTEN, phosphatase and tensin homolog; shRNA, short hairpin RNA; TKI, tyrosine kinase inhibitor; WT/T790M, WT EGFR with a T790M mutation.

Conflict of interest: Pasi A. Jänne and Bruce E. Johnson are part of a pending patent application on EGFR mutations

Citation for this article: J. Clin. Invest. 116:2695-2706 (2006). doi:10.1172/JCI28656
TKIs $(8,15,16)$. Although other cell lines (e.g., breast cancer cell lines) have been used as model systems to investigate sensitivity to gefitinib, the EGFR mutated and amplified lung cancer cell lines used in this study are greater than 10 - to 100 -fold more sensitive to gefitinib $\left(\mathrm{IC}_{50}, \sim 10-100 \mathrm{nM}\right)$ than other cell lines and serve as faithful in vitro models for the lung cancers with the most dramatic clinical responses to EGFR inhibitors (8, 15-19).

Acquired resistance to gefitinib occurs in NSCLC patients with somatic activating mutations in EGFR, who initially respond clinically to treatment with gefitinib but then develop progressive cancer. This acquired resistance has been associated with the development of a secondary mutation in EGFR analogous to those observed in $B C R-A b l$ and KIT in imatinib-resistant chronic myelogenous leukemia and gastrointestinal stromal cell tumors, respectively $(20,21)$. Initial studies have identified a secondary EGFR mutation, T790M, in NSCLC tumor biopsies from 4 of 8 individuals who developed disease progression while receiving EGFR TKI treatment (22-24). The T790M mutation is believed to abrogate gefitinib's ability to bind and inhibit the EGFR. When EGFR T790M alone or cis to an activating EGFR mutation is transfected into Cos-7 or Ba/F3 cells, the EGFR autophosphorylation is resistant to inhibition by gefitinib $(24,25)$. However, it remains unknown whether acquisition of T790M alone is sufficient to make a gefitinib-sensitive EGFR mutant NSCLC resistant to gefitinib-induced cell death. Additionally, the importance of whether T790M occurs cis or trans to the somatic activating EGFR mutation in gefitinib-resistant tumors remains to 

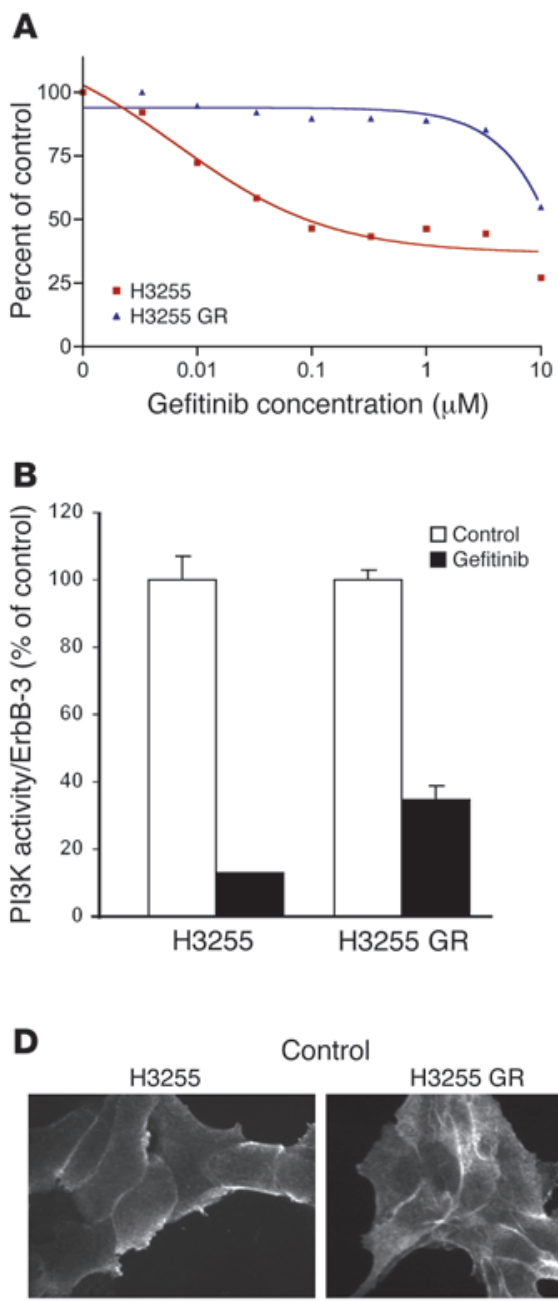

Control H3255 GR
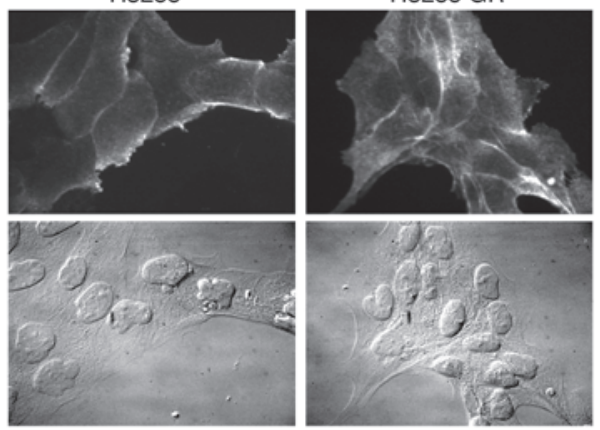
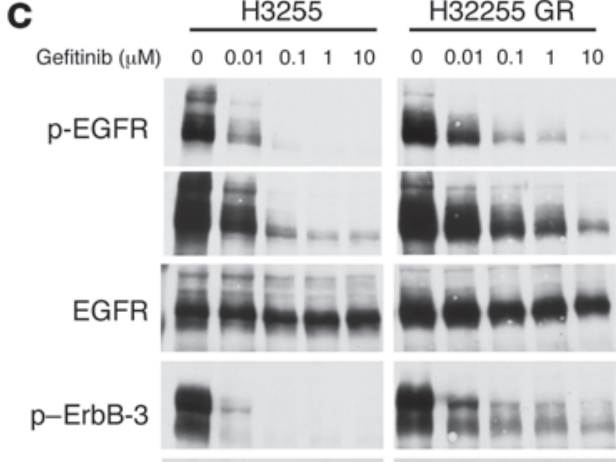

\section{ErbB-3}
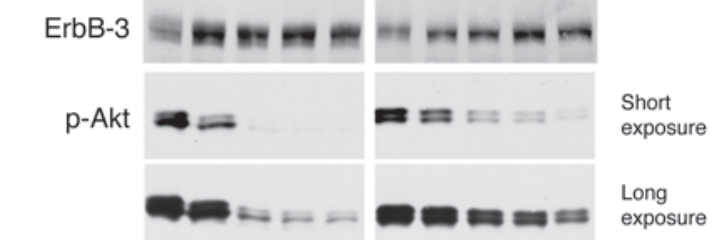

Figure 1

Generation of a cell line, H3255 GR, with acquired resistance to gefitinib. (A) H3255 and H3255 GR cell lines were subjected to MTS survival assays in increasing concentrations of gefitinib (see Methods). (B) ErbB-3 was immunoprecipitated from H3255 and H3255 GR cells grown with or without $1 \mu \mathrm{M}$ gefitinib for 6 hours. Half of the immunoprecipitate was used for ErbB-3 quantification; the other half was used for PI3K assays (see Methods). PI3K activity normalized to ErbB-3 protein is shown as a percent of control (no gefitinib). (C) H3255 and H3255 GR cells were exposed to increasing amounts of gefitinib for 24 hours prior to lysis. Blots of H3255 and H3255 GR extracts for each antibody are the same exposure from a single gel and blot; an irrelevant lane between the H3255 and H3255 GR extracts was omitted. (D) H3255 and H3255 GR cells were serum-starved overnight followed by treatment with or without $1 \mu \mathrm{M}$ gefitinib for 3 hours followed by stimulation with EGF. Immunofluorescence was performed using anti-p-EGFR (pY1068) antibodies. Left: p-EGFR membrane staining by immunofluorescence in untreated H3255 and H3255 GR cells and corresponding phase-contrast images (bottom panels). Right: Immunofluorescence and phase-contrast images of H3255 and H3255 GR cells treated with gefitinib. Most H3255 GR cells maintained detectable membrane EGFR staining in the presence of gefitinib (arrows). The gefitinib-treated panels were equally overexposed to highlight the residual membrane staining. Magnification, $\times 60$. more, the clinical significance, if any, of rare T790M sequences is not known.

The in vitro sensitivity of NSCLC cell lines to EGFR TKI treatment is closely correlated with downregulation of the PI3K/Akt pathway $(17,26)$. Moreover, in a previous study we demonstrated that NSCLC cell lines sensitive to gefitinib are distinct in that they use ErbB-3 to activate the PI3K/ Akt pathway (26). In fact, preliminary studies demonstrate that ErbB-3 protein expression is associated with efficacy of EGFR TKI therapy in patients with NSCLC (27). However, it is unknown whether downregulation of ErbB-3/PI3K/Akt signaling correlates with sensitivity to gefitinib or if it is necessary for gefitinib to promote cell death.

In this study, we model the development of acquired resistance to gefitinib in patients with NSCLC by generating a gefitinib-resistant H3255 (H3255 GR) cell line in vitro. This cell line acquires a T790M mutation only in a small fraction of the amplified EGFR alleles. The T790M allele is undetectable by conventional sequencing and requires a highly sensitive HPLC-based technique for its detection. We found that exogenous introduction of $E G F R$ $\mathrm{T} 790 \mathrm{M}$ conferred resistance to gefitinib-induced cell death in vitro and in vivo when in cis to an activating mutation. We further demonstrated that continued activation of the PI3K/Akt pathway by EGFR-independent mechanisms was sufficient to confer resistance to gefitinib in these EGFR mutant and amplified lung cancers. These observations indicate that restoration of PI3K activation via either a rare T790M mutation or other mechanisms can provide resistance to gefitinib. be determined. Moreover, some acquired-resistance tumors have been shown to harbor a very low percentage of T790M-containing sequences $(22,23)$. The mechanism by which a small proportion of T790M sequences confers resistance remains undefined. Further-

\section{Results}

Development of H3255 GR, a gefitinib-resistant NSCLC cell line. Despite the clinical and radiographic benefits of gefitinib and erlotinib treatment in many patients with activating EGFR mutations, 
Table 1

Summary of parental and engineered cell lines

\begin{tabular}{lcccc} 
Cell line & $\begin{array}{c}\text { EGFR } \\
\text { mutation }\end{array}$ & $\begin{array}{c}\text { Expression } \\
\text { construct }\end{array}$ & $\begin{array}{c}\text { IC }_{50} \text {, gefitinib } \\
(\mu \mathbf{M})\end{array}$ & $\begin{array}{c}\text { IC } 50, \text { CL-387,785 } \\
(\mu \mathbf{M})\end{array}$ \\
$\begin{array}{l}\text { Parental } \\
\text { H3255 }\end{array}$ & L858R & NA & 0.08 & 0.085 \\
H3255 GR & L858R/T790M & NA & $>10$ & 5.56 \\
H1975 & L858R/T790M & NA & $>10$ & 2.26 \\
HCC827 & Del E746_A750 & NA & 0.008 & 0.002 \\
Engineered & & & & \\
H3255 GFP & L858R & GFP & 0.12 & 0.024 \\
H3255 WT/T790M & L858R & WT/T790M & $>10$ & 0.14 \\
H3255 L858R/T790M & L858R & L858R/T790M & $>10$ & 1.17 \\
H3255 Del/T790M & L858R & Del/T790M & $>10$ & 1.06 \\
HCC827 GFP & Del E746_A750 & GFP & 0.002 & 0.002 \\
HCC827 WT/T790M & Del E746_A750 & WT/T790M & 0.003 & 0.002 \\
HCC827 L858R/T790M & Del E746_A750 & L858R/T790M & 4.7 & 0.007 \\
HCC827 Del/T790M & Del E746_A750 & Del/T790M & 4.6 & 0.009 \\
HCC827 p110 $\alpha$ E545K & Del E746_A750 & p110 $\alpha$ E545K & 1.0 & ND \\
HCC827 ca-Akt & Del E746_A750 & ca-Akt & $>10$ & ND \\
\hline
\end{tabular}

ND, not done.

these patients ultimately develop resistance to these agents. In order to model the mechanisms of acquired resistance to gefitinib in vitro, we exposed the gefitinib-sensitive H3255 cell line (EGFR L858R; amplified with $>40$ copies) to increasing concentrations of gefitinib using previously established methods (28). H3255 cells were exposed to serially increasing concentrations of gefitinib for 6 months until the cells were able to proliferate in $100 \mathrm{nM}$ gefitinib with kinetics similar to those of untreated cells (see Methods). We confirmed by 3-(4,5-dimethylthiazol-2-yl)-5-(3-carboxymethoxyphenyl)-2-(4-sulfophenyl)-2H-tetrazolium (MTS) assay that this new cell line, H3255 GR, was not growth inhibited by exposure to gefitinib in vitro (Figure 1A). This cell line was serially passed in gefitinib-free media for more than 15 passages without a change in its sensitivity to gefitinib (data not shown).

We had previously found that the subset of NSCLC cell lines that are sensitive to submicromolar concentrations of gefitinib downregulate PI3K signaling by disrupting its association with ErbB-3 (26). We observed that the H3255 GR cell line maintained ErbB-3-associated PI3K activity to a greater degree than the parental H3255 cells in the presence of gefitinib (Figure 1B). PI3K assays were performed on ErbB-3 immunoprecipitations from both H3255 and H3255 GR cell lines following treatment with or without $1 \mu \mathrm{M}$ gefitinib (Figure 1B). We and others have used $1 \mu \mathrm{M}$ gefitinib in prior studies, as this is reflective of mean steady-state plasma concentrations $(0.4-1.4 \mu \mathrm{M})$ in patients receiving $250 \mathrm{mg}$ gefitinib as determined by phase I clinical studies, and gefitinib begins to have significant offtarget effects at concentrations greater than $1 \mu \mathrm{M}(16,26,29-33)$. Although PI3K activity was inhibited in both cell lines following treatment with gefitinib, the H3255 GR cell line retained approximately 3-fold greater ErbB-3-associated PI3K activity (35\% of control) compared with the parental H3255 cell line (13\% of control).

To determine the mechanism by which H3255 GR cells maintain ErbB-3/PI3K activity despite treatment with gefitinib, we examined the effects of gefitinib treatment on phosphorylation of EGFR, ErbB-3, and downstream signaling molecules (Figure 1C). Unlike the parental H3255 cell line, H3255 GR cells maintained a low level of EGFR phosphorylation even following treatment with $10 \mu \mathrm{M}$ gefitinib (Figure 1C). This subtle but reproducible maintenance of phosphorylated EGFR (p-EGFR) in H3255 GR was consistently observed in several independent experiments (data not shown). Similarly, phosphorylation of ErbB-3, Akt, and ERK1/2 was maintained in the H3255 GR cell line (Figure $1 C)$. Upon exposure to $1 \mu \mathrm{M}$ gefitinib, p-EGFR, p-ErbB-3, and p-Akt persisted at levels approximately $10 \%$ of the untreated controls in the H3255 GR cells, but were only approximately $1 \%$ of the untreated controls in the parental H3255 cells (see Supplemental Figure 1; supplemental material available online with this article; doi:10.1172/ JCI28656DS1.). The H3255 GR cells, unlike the parental H3255 cells, also maintained low levels of p-EGFR staining at the membrane in the presence of $1 \mu \mathrm{M}$ gefitinib (Figure 1D). The H3255 GR cell line also retained phosphatase and tensin homolog (PTEN) expression as determined by Western blotting (data not shown).

H3255 GR cells acquire an EGFR T790M mutation. The above findings suggested that gefitinib is unable to completely inhibit EGFR phosphorylation in the H3255 GR cell line. In light of recent observations that the presence of an EGFR T790M mutation is associated with acquired resistance to EGFR TKIs in patients $(24,25)$, the entire EGFR gene from $\mathrm{H} 3255 \mathrm{GR}$ was sequenced, and no changes from the parental cell line were identified (data not shown). However, the EGFR gene is known to be amplified at least 20-fold (i.e., > 40 copies) in H3255 cells, with preferential amplification of the mutant L858R-containing allele $(15,16)$. Thus, the H3255 GR could possess a resistant allele only in a fraction of the total EGFR alleles that would be undetected by direct sequencing. Therefore, we employed a more sensitive technique using SURVEYOR, a DNA endonuclease that cleaves DNA heteroduplexes 3' of a mismatched site. HPLC separation of the DNA fragments was used to screen for low-frequency genetic alterations in H3255 GR. Our previous studies using SURVEYOR demonstrated that this method can detect activating EGFR mutations even if the mutant allele comprises only $1 \%-5 \%$ of the total alleles (34). We amplified exon 20 from H3255, H1975 (a cell line with an endogenous T790M mutation; see Table 1), and H3255 GR, treated the PCR products with the SURVEYOR endonuclease, and separated the digested products using HPLC (Figure 2A). H1975 is heterozygous for a SNP at position 787 (Q787Q) and for T790M, and thus SURVEYOR digestion produced fragments that migrated as a doublet peak (153 and 154 bps; Figure 2A). These peaks were not observed in $\mathrm{H} 3255$, which is homozygous for the SNP and has no detectable T790M. However, in H3255 GR, a small peak was observed at $154 \mathrm{bp}$ following SURVEYOR treatment, suggesting the presence of a new genetic alteration in exon 20 in this cell line. Using denaturing HPLC (DHPLC) and fractionation, we enriched for the fractions containing the altered allele and sequenced the fractions using previously described methods (34). The sequence tracings from H3255 GR demonstrated the presence of a small population of T790M containing alleles in this cell line (Figure 2B). 
A
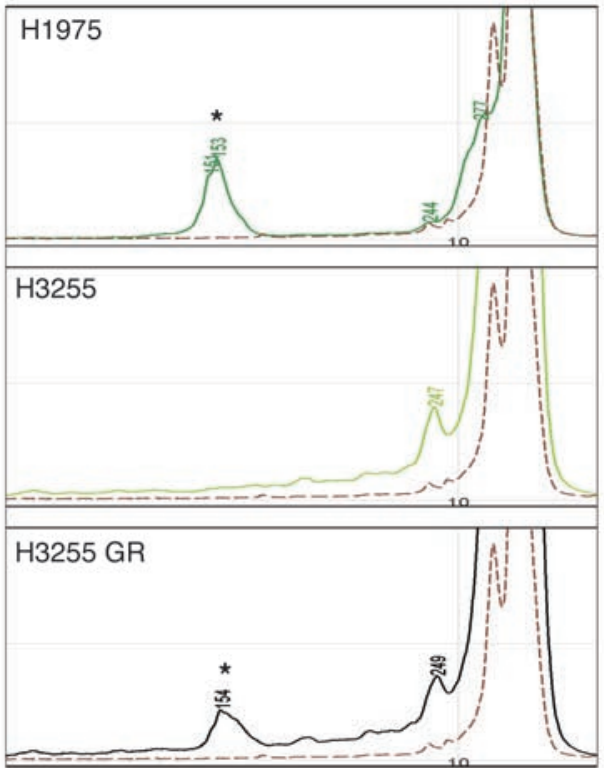

C

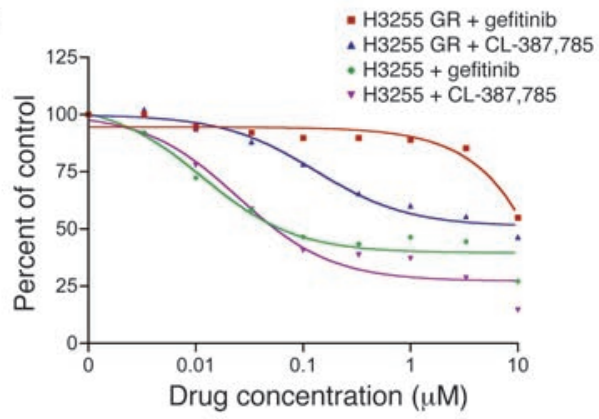

E

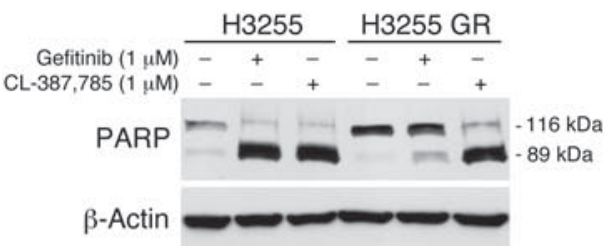

$\mathbf{F}$

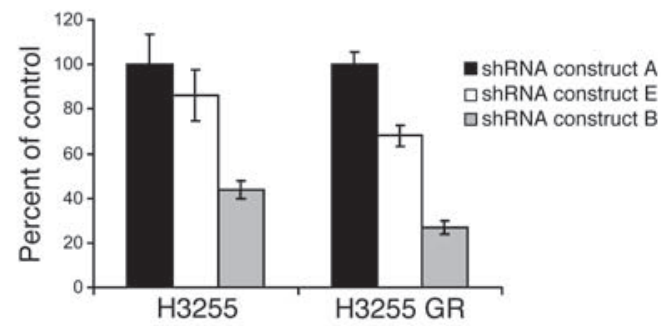

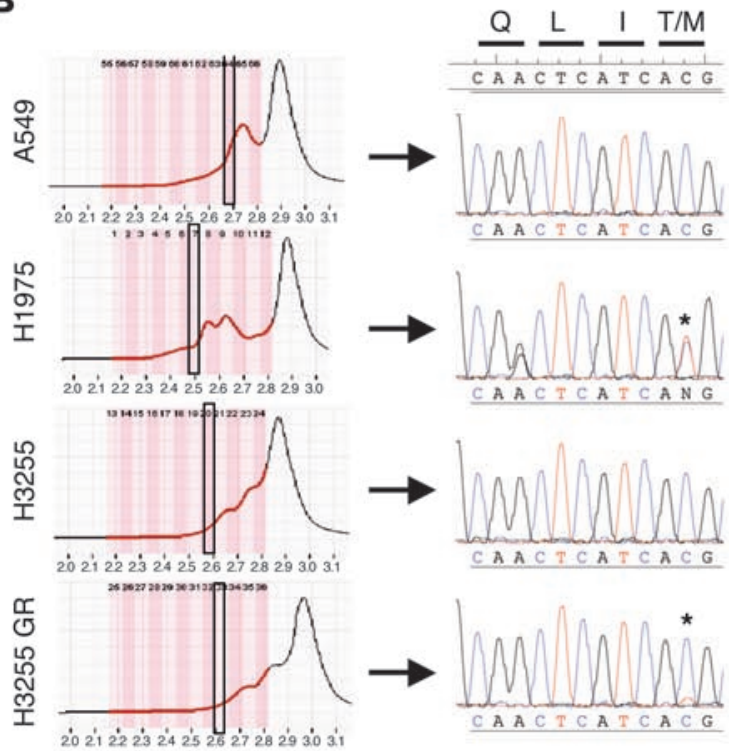

D

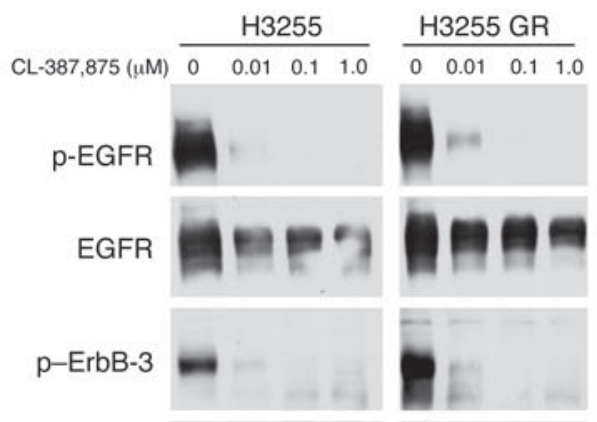

ErbB-3
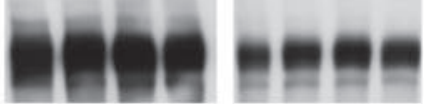

p-Akt
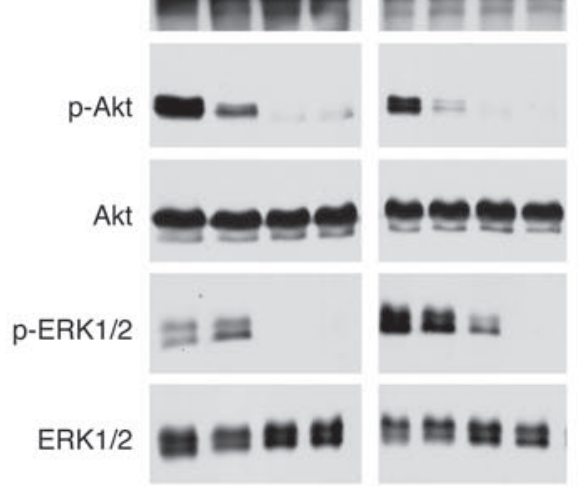

Figure 2

H3255 GR harbors a T790M mutation and is growth-inhibited by an irreversible EGFR inhibitor (CL-387,785) and EGFR-specific shRNA. (A) Exon 20 was amplified by PCR analysis, subjected to SURVEYOR analysis, and analyzed using the WAVE system (see Methods). Shown are HPLC tracings; numbers represent fragment size. Note the presence of a 154-bp fragment in H3255 GR, suggesting a T790M mutation (asterisks), as observed in the H1975 cells (positive control). A549 (dashed line) is shown as a negative control. (B) Exon 20 was amplified from A549 (negative control), $\mathrm{H} 1975$ (positive control), H3255, and H3255 GR cells. Products were separated at a partially denaturing temperature using the WAVE system. The indicated fractions (boxed areas) were directly sequenced (see Methods). The T790M mutation was observed in H3255 GR and $\mathrm{H} 1975$ cells (asterisks). (C) H3255 and H3255 GR cells were treated with increasing concentrations of CL-387,785 or gefitinib and subjected to an MTS survival assay. (D) Western blot analysis of H3255 and H3255 GR cells treated with increasing concentrations of CL-387,785. Blots of H3255 and H3255 GR extracts for each antibody are the same exposure from a single gel and blot; irrelevant lanes between the H3255 and H3255 GR extracts were omitted. (E) Western blot for PARP with H3255 and H3255 GR cells following treatment with gefitinib or CL-387,785 for 72 hours. (F) Lentiviral constructs containing EGFR shRNAs were infected into H3255 and H3255 GR cells, followed by an MTS assay. Growth was normalized to shRNA construct A (control), which did not cause significant EGFR downregulation (Supplemental Figure 4). 
SURVEYOR was also able to detect this mutation in H3255 GR cDNA (Supplemental Figure 2). Using RT-PCR, we amplified exons 20 and 21 from H3255 GR and cloned the PCR products into PCR Blunt (Invitrogen), and 96 individual cDNA clones were sequenced. The T790M allele was the cDNA in 3.3\% (3 of 91) of clones analyzed (Supplemental Figure 3). Using an enrichment strategy and screening with SURVEYOR, we verified the T790M mutation in 3 additional cDNA clones from $\mathrm{H} 3255 \mathrm{GR}$.

Although the methodology used to produce this cell line and the stability of its resistance pattern to gefitinib after more than 15 passages in gefitinib-free media suggests a homogenous population, it remained to be determined whether the mutation was present in all cells in the H3255 GR cell line. Thus we attempted to subclone single cells but were unsuccessful despite culturing in a variety of conditions, including conditioned media. As an alternative approach, we performed PCR on 2 H3255 GR cells followed by SURVEYOR analyses (PCR was unsuccessful from single cells). Analyses of 2 cell PCR products from 8 independent pairs clearly demonstrated the T790M in 7 of 8 PCR products (88\%). These findings suggest that in the H3255 GR cell line most, if not all, cells harbor a T790Mcontaining allele that is present on a small percent of the amplified alleles. This is consistent with the observation that most cells in the H3255 GR population clearly had persistent phosphorylation of the EGFR in the presence of gefitinib (Figure 1D).

The irreversible EGFR inbibitor CL-387,785 effectively inhibits the growth of H3255 GR cells. We examined the efficacy of CL-387,785 against H3255 GR cells, as this compound is more effective than gefitinib at inhibiting the EGFR T790M mutant $(23,24)$. We found that CL-387,785 was more potent than gefitinib at inhibiting the growth of the H3255 GR cell line, while its effect on the parental cell line was similar to that of gefitinib (Figure 2C). Although CL-387,785 is effective against EGFR T790M, it inhibits non-T790M-containing EGFR more potently (24). This is consistent with its greater activity against $\mathrm{H} 3255$ compared with H3255 GR. CL-387,785 also inhibited the growth of $\mathrm{H} 1975$ cells (Table 1), results consistent with prior studies (25). CL-387,785 had no effect on EGFR WT cell lines known to be resistant to gefitinib (A549 and H441; $\mathrm{IC}_{50},>10$ $\mu \mathrm{M}$; data not shown). In H3255 GR cells, the phosphorylation of EGFR and ErbB-3 was completely inhibited with concentrations of at least $100 \mathrm{nM}$ CL-387,785, unlike with gefitinib treatment, (Figure 2D). Furthermore, at the same concentration of $100 \mathrm{nM}$, substantial downregulation of Akt and ERK1/2 phosphorylation was observed in both the parental and the gefitinib-resistant cell lines. This supports the notion that Akt and ERK signaling remain under the control of EGFR (via ErbB-3 for Akt) in H3255 GR cells. Consistent with these observations, CL-387,785, but not gefitinib, induced marked apoptosis in the H3255 GR cell line (as indicated by an increase in the $89-\mathrm{kDa}$ cleaved product of poly ADP-ribose polymerase [PARP]), while both agents induced apoptosis in the parental H3255 cell line (Figure 2E). These results are consistent with the notion that acquisition of the T790M allele is the major resistance mechanism in this cell line.

H3255 GR cells maintain EGFR dependence. The observed efficacy of CL-387,785 against H3255 GR cells could be attributed to non-EGFR mechanism of action of this agent. In order to determine whether H3255 GR was still dependent on EGFR for growth, we downregulated EGFR expression using short hairpin RNAs (shRNAs). Six different lentiviral EGFR shRNA constructs were infected into A549 cells, which are not dependent on EGFR for their growth, and EGFR expression was determined (Supplemental Figure 4). shRNA con- struct B reproducibly caused the greatest EGFR downregulation, while shRNAs A and E led to no or minimal EGFR downregulation (Supplemental Figure 4). The shRNA constructs were infected into H3255 and H3255 GR cells, and growth was assayed 7 days later using an MTS assay (Figure 2F). shRNA construct B led to significant growth inhibition in both H3255 and H3255 GR cells $(P<0.001$; paired Student's $t$ test) compared with the control shRNA construct A. These findings support the notion of continued EGFR dependence in the gefitinib-resistant H3255 GR cell line. We also generated 6 additional shRNA constructs in order to specifically downregulate the T790M-containing allele. However, none of the constructs effectively downregulated EGFR T790M (data not shown).

EGFR T790M confers resistance to gefitinib more effectively when cis to an activating L858R or exon 19 deletion mutation. Both H3255 GR and H1975 cells contained the EGFR T790M mutation and displayed resistance to gefitinib. However, these studies did not address whether EGFR T790M is the sole mechanism of resistance in these cell lines or whether expression of a T790M mutant of EGFR is sufficient to confer a resistant phenotype to a gefitinib-sensitive NSCLC cell line. Thus, we retrovirally infected HCC827 and H3255 cell lines to stably express WT EGFR with a T790M mutation (WT/T790M) or EGFR with a T790M and an activating mutation (i.e., T790M cis to an L858R mutation [L858R/T790M] or an exon 19 deletion mutation [Del/T790M]; see Methods and Table 1). Total EGFR expression, as measured by quantitative RT-PCR, was increased by 1.3 - to 2 -fold compared with GFP-infected H3255 or HCC827 cells (Supplemental Table 1). Using both direct sequencing and SURVEYOR, we detected EGFR T790M in all of the cell lines. HCC827 cells infected with either GFP control or WT/T790M remained remarkably sensitive to gefitinib (Figure $3 \mathrm{~A}$ ). In contrast, expression of the double mutants L858R/T790M and Del/T790M rendered HCC827 cells resistant to gefitinib (Figure 3A), even though they were expressed at lower levels than WT/T790M (Supplemental Table 1). In fact, direct sequencing revealed that the T790M was a minor but detectable allele in these 2 double-mutant cell lines, consistent with the modest increase in total EGFR expression (Supplemental Table 1 and data not shown). Notably, resistance was promoted by the presence of a different activating EGFR mutation, L858R/T790M, even though the HCC827 cell line contains an EGFR exon 19 deletion (E746_A750; ref. 15). Similarly, stable expression of L858R/T790M or Del/T790M in H3255 rendered this cell line resistant to gefitinib (Figure 3B). Interestingly, although WT/T790M did not cause gefitinib resistance in HCC827 cells, it promoted partial gefitinib resistance - albeit to a lesser extent than when combined with an activating mutation - in H3255 cells. These results were observed in cells derived from 3 independent infections. Gefitinib treatment of HCC827 GFP and HCC827 WT/T790M led to similar inhibition of EGFR, ErbB-3, Akt, and ERK1/2 phosphorylation, with almost complete inhibition observed at concentrations greater than $10 \mathrm{nM}$ (Figure 3C). In contrast, in both HCC827 L858R/T790M and HCC827 Del/T790M, gefitinib (up to $1 \mu \mathrm{M}$ ) was unable to inhibit EGFR phosphorylation and caused only minimal decreases in p-ErbB-3, p-Akt, and p-ERK1/2 (Figure 3C). This is consistent with the notion that the activating mutant EGFRs are more potent signaling proteins than WT EGFR, and thus when resistant to the effects of gefitinib by coexisting T790M mutations, they provide a greater oncogenic stimulus. In H3255 cells, gefitinib was able to partially inhibit p-EGFR, p-ErbB-3, and p-Akt in H3255 WT/T790M cells, while it had no effect on these signaling proteins in $\mathrm{H} 3255$ L858R/T790M or H3255 Del/T790M cells (Supplemental Figure 5). 

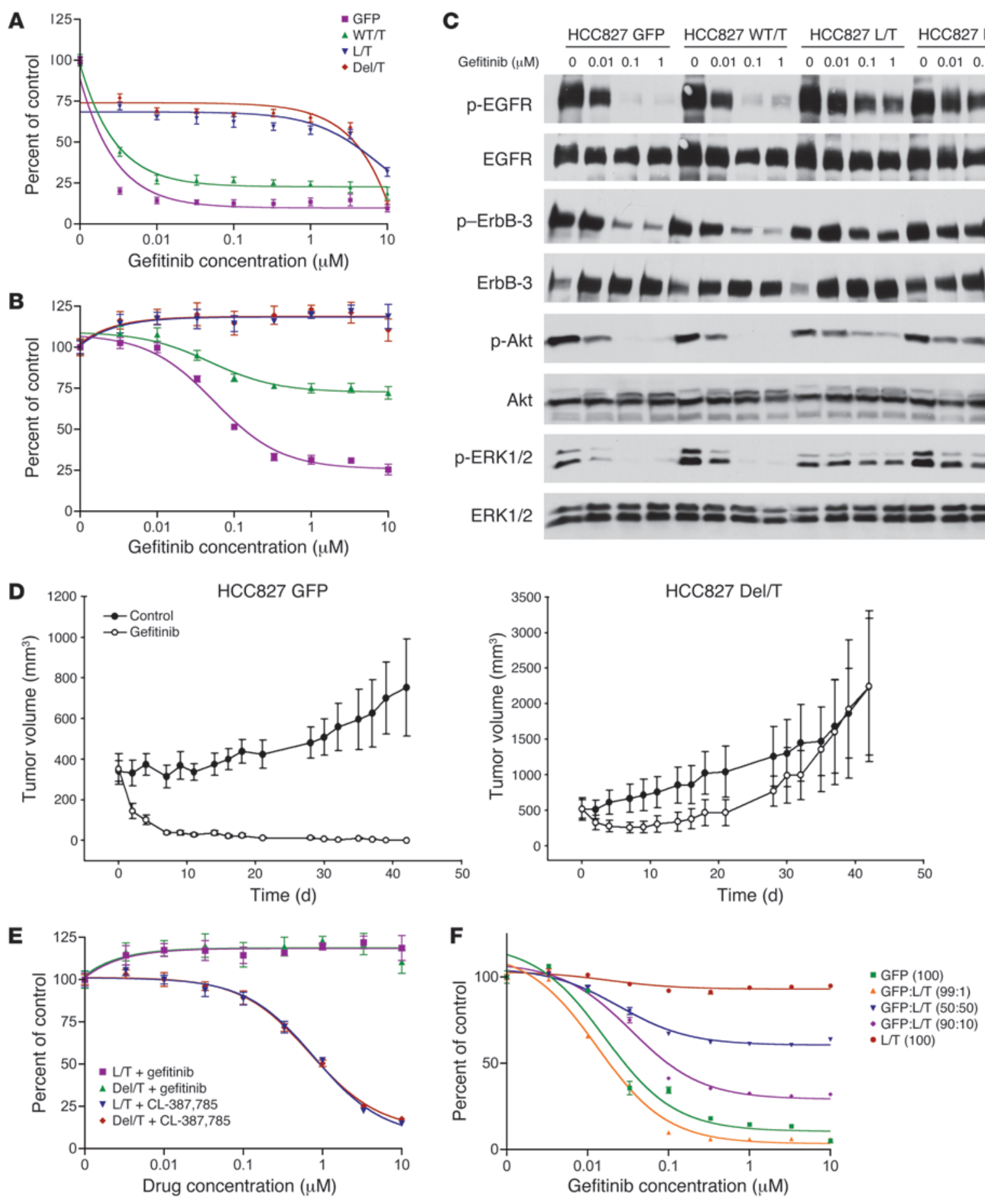
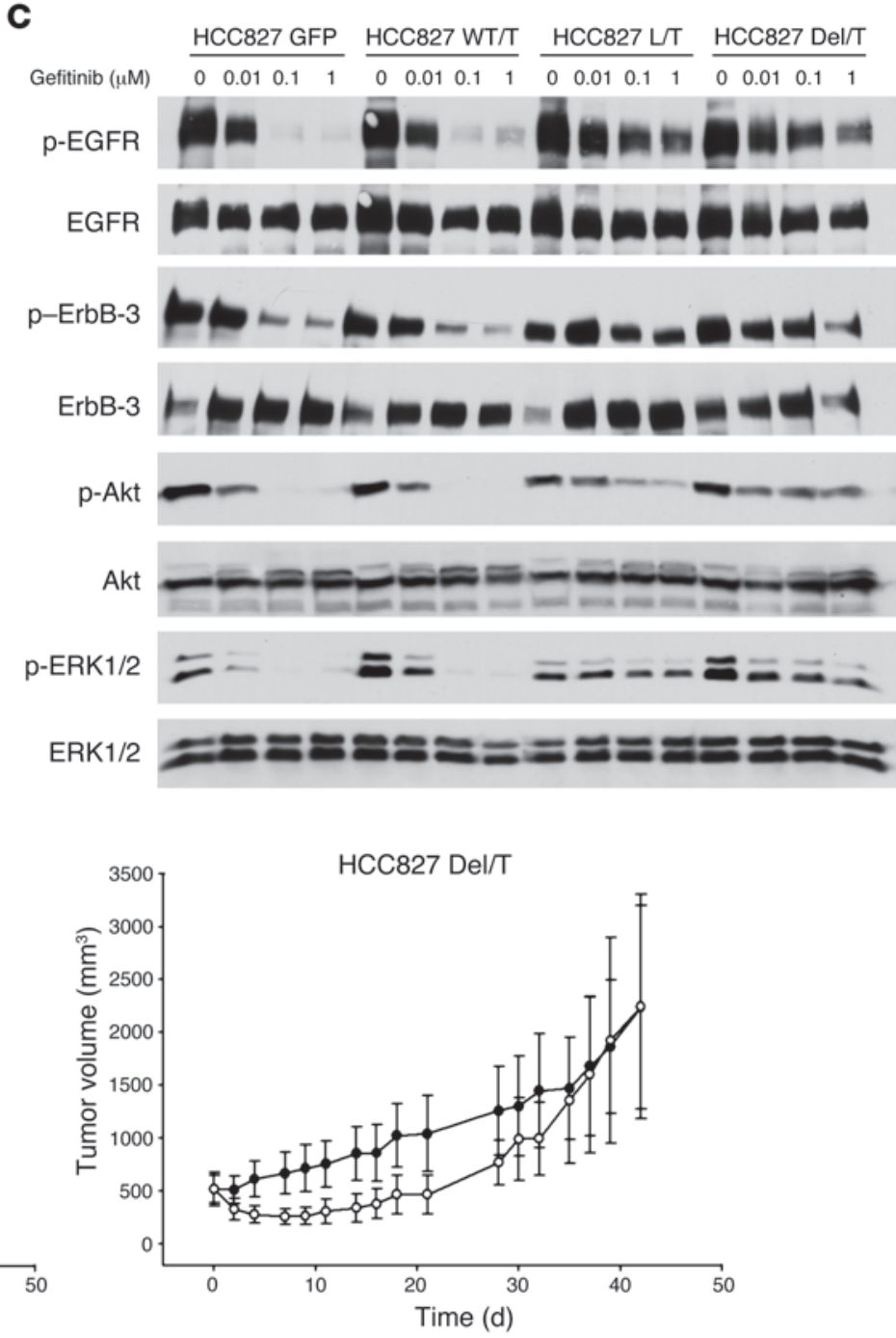

\section{Figure 3}

Expression of EGFR with a L858R/T790M or Del/T790M mutation more effectively promotes gefitinib resistance in HCC827 and H3255 cells. (A) The single-mutant WT/T790M (WT/T), the double-mutant L858R/T790M (L/T) and Del/T790M (Del/T), and GFP (as a control) were introduced into HCC827 cells. The resulting cell lines were subjected to an MTS survival assay in increasing concentrations of gefitinib. (B) The same retroviruses as in A were used to infect $\mathrm{H} 3255$ cells, and resulting cell lines were subjected to MTS survival assays in increasing doses of gefitinib. (C) Lysates from HCC827 cells expressing EGFR mutants or GFP control and exposed to increasing concentrations of gefitinib for 12 hours were probed with the indicated antibodies. (D) Xenografts in nu/nu mice were generated from either HCC827 Del/T790M or HCC827 GFP cells. Gefitinib was administered by oral gavage, and tumors were measured 3 times weekly. Mean tumor volumes are shown. (E) The H3255 cell lines were subjected to an MTS survival assay in the presence of increasing concentrations of CL-387,785 and gefitinib. (F) H3255 L858R/T790M and H3255 GFP cells were seeded together at different ratios to determine whether a minor percent of L858R/T790M cells could confer resistance to cells with no T790M mutation. Cells were seeded in the indicated ratios (in parentheses) of H3255 GFP cells to H3255 L858R/T790M cells and subjected to an MTS survival assay. 
These observations are consistent with gefitinib's effects on growth in these cell lines (Figure 3B).

Our observations from exogenous introduction of T790M into H3255 and HCC827 cells suggest that EGFR T790M more potently promotes resistance to gefitinib when present cis to the EGFRactivating mutation. In order to determine whether H1975 and H3255 GR also contain the T790M allele in cis to the activating mutation, we performed RT-PCR and sequenced clones from both cell lines. In both cell lines the T790M allele was only found cis to the L858R allele (6 of 6 independent clones in H3255 GR; 21 of 21 independent clones in H1975).

We also generated xenografts in $n u / n u$ mice with HCC827 GFP and HCC827 Del/T790M cell lines and treated these mice with gefitinib (Figure 3D). H3255 did not grow as a xenograft (data not shown). Gefitinib treatment resulted in complete regression (as detected by palpation) of tumors in 5 of 5 HCC827 GFP mice (Figure 3D and Supplemental Figure 6). In contrast, HCC827 Del/T790M tumors were resistant to gefitinib. The comparison between HCC827 GFP and HCC827 Del/T790M growth patterns in mice treated with gefitinib was highly statistically significant $(P=0.0006)$.

Because the irreversible inhibitor CL-387,785 retains some activity against EGFR T790M, the cell lines stably expressing the T790Mcontaining constructs were exposed to CL-387,785 and subjected to an MTS survival assay. CL-387,785 treatment inhibited survival of both H3255 L858R/T790M and H3255 Del/T790M cells. Both H3255 GFP and H3255 WT/T790M were growth inhibited at low concentrations of CL-387,785 (Table 1). Similar findings were observed for analogous studies performed on the HCC827 cell lines treated with CL-387,785 (Supplemental Figure 7A and Table 1).

EGFR T790M does not confer resistance to surrounding cells via a paracrine effect. Previous studies have suggested that some EGFR TKIresistant tumors contain a low percentage of T790M-containing cells (23), leading some to speculate that a small percent of resistant cells may confer resistance to the neighboring cells, perhaps via a paracrine effect. To formally test this hypothesis, we sought to determine whether the in vitro resistant H3255 L858R/T790M cells protect sensitive $\mathrm{H} 3255 \mathrm{GFP}$ cells from the effects of gefitinib. We generated mixtures of H3255 GFP with H3255 L858R/T790M in different ratios and examined the effects on growth using the MTS assay. As shown in Figure 3F, the cultures consisting of $10 \%$ and $50 \%$ of the L858R/T790M cells demonstrated increasing levels of intermediate resistance, whereas the control cultures of $100 \%$ GFP cells remained sensitive and those of $100 \%$ L858R/T790M cells remained resistant. These results suggest that there is no significant protection of non-T790M-containing cells by cocultured cells harboring a resistant T790M-containing clone. Similar findings were observed for the analogous studies performed on the HCC827 cell lines (Supplemental Figure 7B).

Oncogenic activation of the $P I 3 K / A k t$ pathway confers resistance to gefitinib. In this and our previous study, we have observed that downregulation of the PI3K/Akt pathway correlates with sensitivity to gefitinib in NSCLC cell lines (26). Although we have demonstrated that an EGFR T790M mutation (when in cis to an EGFR-activating mutation) confers gefitinib resistance and maintains PI3K/Akt signaling, it remains unknown whether continued PI3K/Akt signaling alone is sufficient to make these cancers resistant to gefitinib. Somatic mutations in the PIK3CA gene, which encodes for the p110 $\alpha$ catalytic subunit of class IA PI3Ks, have been identified in approximately $5 \%$ of NSCLCs (35). We and others have shown that these mutations result in sustained Akt activation under serum- deprived conditions (35-38). To determine whether downregulation of PI3K/Akt signaling is necessary for an EGFR mutant NSCLC cell to be sensitive to gefitinib, we introduced the p $110 \alpha \mathrm{E} 545 \mathrm{~K}$ into HCC827 cells via retroviral infection. HCC827 cell lines harboring activated PI3K (HCC827 p110 $\alpha$ E545K cells) were more resistant to gefitinib in vitro $\left(\mathrm{IC}_{50}, 1 \mu \mathrm{M}\right)$ compared with those harboring GFP alone $\left(\mathrm{IC}_{50}, 0.01 \mu \mathrm{M}\right.$; Figure $4 \mathrm{~A}$ and Table 1$)$. The presence of $\mathrm{p} 110 \alpha$ E545K also protected HCC827 cells from gefitinib-mediated apoptosis, as assessed by either flow cytometry or PARP cleavage (Figure 4, B and C). As shown in Figure 4D, the oncogenic p110 $\alpha$ E545K maintained some Akt phosphorylation in the presence of gefitinib (35\% of untreated GFP cells and 10\% of untreated p $110 \alpha$ E545K cells). Additionally, we observed that expression of constitutively active Akt (ca-Akt) also conferred resistance to gefitinib-mediated cell killing (Figure 4, A and E). Therefore, continued activation of $\mathrm{PI} 3 \mathrm{~K} /$ Akt signaling is sufficient to confer resistance to gefitinib.

\section{Discussion}

In the present study, we examined mechanisms of resistance to gefitinib in highly sensitive EGFR mutant and amplified NSCLC cell lines. Prior studies suggest that about $50 \%$ of NSCLC patients who initially respond to gefitinib or erlotinib and subsequently develop clinical resistance to EGFR TKIs acquire a detectable secondary T790M mutation in EGFR (22-24). The H3255 GR cell line, a model of acquired resistance to gefitinib, remarkably recapitulated this clinical observation in vitro, as the T790M mutation was detected only in this cell line, not in the parental H3255 cell line. A surprising observation from our studies was the dominant effect of a low fraction of T790M-containing alleles in the H3255 GR cell line. H3255 is amplified more than 20-fold at the EGFR locus. Gefitinib treatment continued to inhibit the majority of EGFR alleles in H3255 GR cells, as demonstrated by the decrease in p-EGFR detected by Western blot analysis and immunofluorescence (Figure 1, C and D). This suggests that the T790M-containing allele(s) can act in a dominant fashion allowing continued maintenance of EGFR, ErbB-3/PI3K/Akt, and ERK1/2 phosphorylation in the H3255 GR cell line and inhibition of apoptosis (Figure 1, B and C, and Figure 2E). Although T790M EGFR is resistant to gefitinib's inhibitor effects, the T790M mutant may also make EGFR a more potent signaling protein. It has previously been demonstrated that there is greater constitutive EGFR autophosphorylation of a T790M mutant EGFR compared with WT EGFR - more specifically, by EGFR L858R/T790M compared with EGFR L858R when expressed in Ba/F3 cells $(25,39)$. Additionally, the recent report that germline EGFR T790M mutations are linked with familial lung cancers underscores the increased activity of the T790M mutant (40). Furthermore, cell lines (such as H1975) and patients with L858R/T790M who never received gefitinib treatment have been described, suggesting an independent, oncogenic role for the T790M mutation (41). These observations may account for the resistance promoted by only a small fraction of EGFR L858R/ T790M alleles in H3255 GR cells. Furthermore, these findings are consistent with the observed differences in gefitinib resistance when T790M was introduced in cis compared with trans with an activating EGFR mutation into HCC827 and $\mathrm{H} 3255$ cell lines.

Since EGFR is amplified in the H3255 cell line, the presence of the secondary mutation in a minority of EGFR alleles in H3255 GR was initially not detected using conventional direct sequencing and was only detectable using a more sensitive enzymatic technique. Our findings suggest that the prevalence of the T790M mutation as a mechanism of resistance to gefitinib and erlotinib may be 

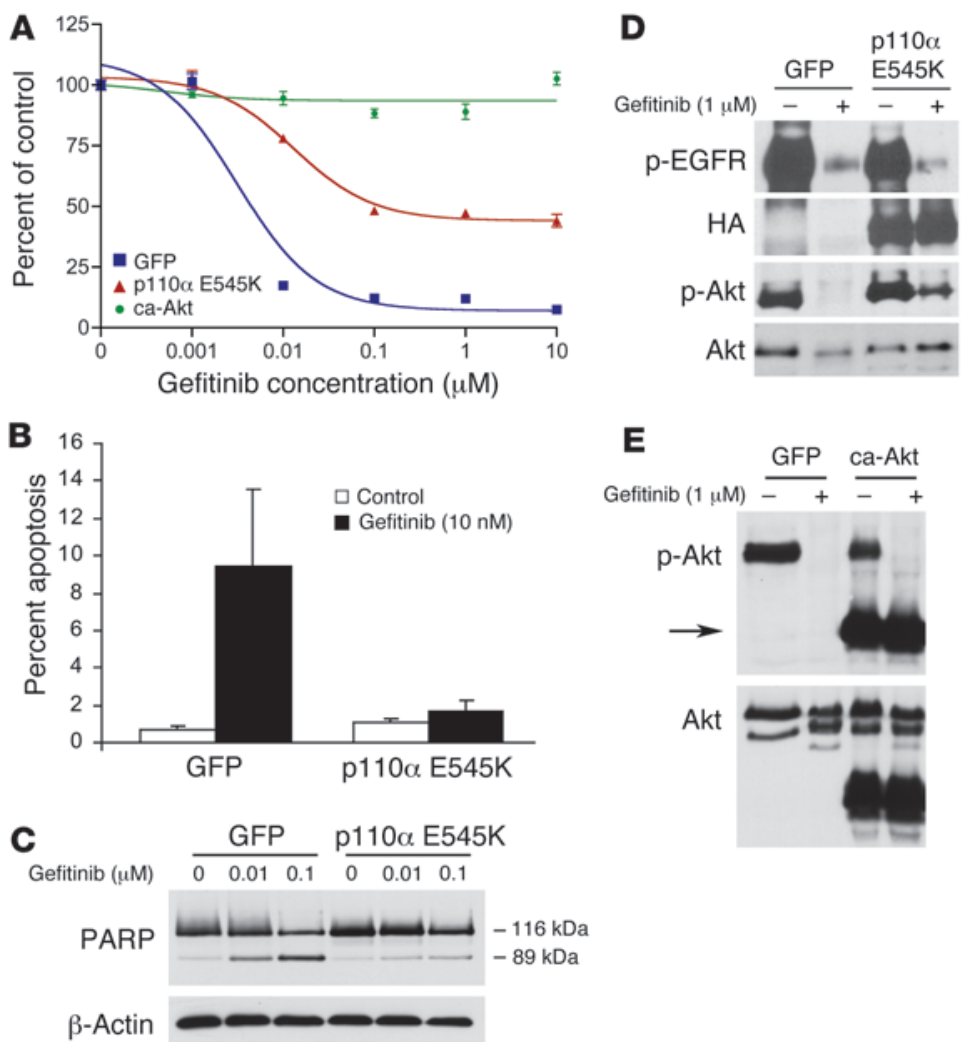

Figure 4

Downregulation of $\mathrm{PI} 3 \mathrm{~K} / \mathrm{Akt}$ signaling is necessary for gefitinib to effectively induce apoptosis. (A) Retroviral expression of HA-tagged oncogenic PI3K (p110 $\alpha$ E545K) or ca-Akt into HCC827 cells conferred resistance to gefitinib. Expression constructs were introduced into HCC827 cells by retroviral infection, and the resulting cell lines were subjected to an MTS survival assay in increasing concentrations of gefitinib. (B) Expression of p110 $\alpha$ E545K abrogated apoptosis induced by gefitinib. HCC827 cells expressing GFP or p110 $\alpha$ E45K were treated with $10 \mathrm{nM}$ gefitinib for 72 hours and assessed for apoptosis by analyzing the percent of cells in SubG1 by FACS analysis as previously described (30). (C) Cells were treated as in B, lysed, and assessed for PARP cleavage. Increasing gefitinib concentrations led to increased appearance of the cleaved $89-\mathrm{kDa}$ fragment in HCC827 GFP cells compared with HCC827 p110 $\alpha$ E545K cells. (D) p110 $\alpha$ E545K led to persistent Akt activation. HCC827 cells expressing GFP or p110 $\alpha$ E545K were exposed to $1 \mu \mathrm{M}$ gefitinib for 12 hours prior to lysis. Note that expression of HA-tagged P110 $\alpha$ E545K led to diminished but persistent p-Akt expression in the presence of gefitinib. (E) ca-Akt was not inhibited by gefitinib. HCC827 ca-Akt and HCC827 GFP cells were grown with or without $1 \mu \mathrm{M}$ gefitinib for 12 hours and lysed for Western blot analysis. Arrow indicates ca-Akt that migrates at a lower molecular weight because it lacks the pleckstrin homology domain. Gefitinib inhibited the phosphorylation of the endogenous but not the myristoylated Akt.

more common than previously described. To this end, we have now identified 3 patients who did not have a T790M mutation detected by direct sequencing in whom SURVEYOR detected the presence of a T790M mutation (Table 2). Two of the patients were treated with gefitinib and had T790M present only in the relapsed tumor specimen, while in the remaining patient, T790M was present in a pretreatment tumor specimen. In patient 3, the T790M mutation was detected only in 2 of 5 independent direct sequencing reactions from the relapsed specimen (Table 2). Therefore, direct sequencing alone is not a sensitive enough method to detect the T790M mutation in patients with NSCLC. The findings in this study suggest that the presence of rare T790M-containing sequences in some gefitinib-resistant NSCLC tumors are due to rare T790Mcontaining alleles in most tumor cells, not to rare T790Mcontaining cells within the tumor (23). We demonstrated that these T790M alleles have clinical significance and are sufficient to confer resistance to gefitinib. To date, the finding of a limited number of resistant alleles in the amplified locus appears unique to EGFR in lung cancers. The majority of EGFR mutant NSCLC tumors and cell lines contain concurrent EGFR amplifications (13-16). In contrast, amplification of KIT or PDGFRA in gastrointestinal stromal cell tumors or $B C R-A b l$ in chronic myelogenous leukemia occurs only in the minority of imatinibresistant patients $(20,21,42-44)$. These findings have potential implications for detecting resistance mutations in other oncogenes associated with amplification, such as $E r b B-2$ in breast cancer and EGFR in glioblastoma multiforme $(45,46)$. Recent studies have used genome-wide sequencing efforts to identify mutations in the entire family of kinases in human cancers $(8,47)$. Our findings suggest that sequencing alone may be insufficient to identify all functionally and clinically significant mutations, such as T790M, and should be combined with more sensitive techniques of mutation detection.

The irreversible EGFR inhibitor CL-387,785, which binds covalently to cys-773 of EGFR, retains some ability to inhibit EGFR containing the T790M mutation, causes growth inhibition and downregulation of the ErbB-3/PI3K/Akt axis, and induces apoptosis in T790Mcontaining H3255 GR. Of note, the H3255 GR cells retained PTEN expression, had not acquired a PIK3CA gene mutation, and demonstrated decreased p-Akt levels and loss of PI3K-ErbB-3 interaction upon exposure to CL-387,785 (Figure 2D and data not shown). These findings are consistent with - but do not absolutely prove - the notion that PI3K/Akt signaling remains under the control of EGFR (via ErbB-3) as in the parental H3255 cells. The acquisition of the T790M allele is likely what confers continued signaling in the presence of gefitinib. We further demonstrated, using oncogenic p $110 \alpha$ E545K and ca-Akt, that downregulation of the PI3K/Akt signaling pathway not only correlated with sensitivity to gefitinib, but was required for gefitinib to promote its proapoptotic effect. Thus, continued activation of the PI3K/Akt pathway either through EGFRdependent (T790M) or -independent mechanisms is sufficient to render an EGFR mutant NSCLC resistant to gefitinib. This distinction has clinical relevance and may assist in guiding therapeutic decisions for lung cancer patients developing acquired resistance to gefitinib. Patients with EGFR-dependent mechanisms of resistance to gefitinib may still clinically benefit from a newer generation of EGFR inhibitors, which are presently undergoing clinical evaluation $(24,25)$. However, those with EGFR-independent mechanisms of resistance may need alternative forms of treatment.

Other recent studies have observed that persistent PI3K signaling confers resistance to ErbB inhibitors in other cellular contexts. For example, the efficacy of the anti-ErbB-2 monocloncal antibody trastuzumab is significantly reduced in patients with ErbB-2-amplified breast cancer that lack PTEN (48). Similarly, EGFR TKIs are ineffective in glioblastoma tumors and cell lines as well as in breast can- 


\section{Table 2}

Summary of NSCLC patients with T790M mutations

\begin{tabular}{|c|c|c|c|c|c|c|}
\hline \multirow{2}{*}{ Patient } & \multirow{2}{*}{ Gefitinib $\mathbf{R}_{\mathbf{x}}$} & \multirow{2}{*}{ Treatment duration } & \multicolumn{2}{|c|}{ Pretreatment EGFR mutation } & \multicolumn{2}{|c|}{ EGFR mutation at disease progression } \\
\hline & & & Sequencing & SURVEYOR/WAVE & Sequencing & SURVEYOR/WAVE \\
\hline 1 & No & NA & WT & WT/T790M & NA & NA \\
\hline 2 & Yes & $15 \mathrm{mo}$ & L747_E749 & L747_E749 & L747_E749 & L747_E749/T790M \\
\hline 3 & Yes & $14 \mathrm{mo}$ & E746_A750 & E746_A750 & E746 A750A & E746_A750/T790M \\
\hline
\end{tabular}

Shown are 3 patients in whom EGFR T790M was detected using the SURVEYOR analyses but undetected by direct sequencing. All specimens in which SURVEYOR indicated a T790M mutation were subjected to DHPLC, fractionation, and sequencing as previously described (32). AT790M was detected by direct sequencing in 2 of 5 independent sequence tracings.

cer cell lines that lack PTEN expression $(18,45)$. Restoration of PTEN expression into PTEN mutant MDA-468 breast cancer cells can sensitize them to gefitinib, and downregulation of PTEN using shRNA in the EGFR TKI-sensitive A431 cells (amplified WT EGFR) reduces the apoptosis induced by either erlotinib or the EGFR/ErbB-2 TKI PKI-166 (45). Our observations that continued PI3K signaling conferred resistance to gefitinib even in these EGFR mutant and amplified lung cancers further strengthens the concept that downregulation of PI3K signaling is necessary for ErbB inhibitors to be effective. Furthermore, this is the first study to our knowledge to observe that the recently identified PIK3CA oncogenes are sufficient to promote continued PI3K signaling in gefitinib-sensitive cancer cells treated with gefitinib. Thus, these studies predict that cancers with PIK3CA oncogenic mutations are unlikely to respond to EGFR inhibitors.

Our experimental findings that in vitro and in vivo resistance was generated by introducing EGFR-activating mutations in cis with the T790M mutation provide several important observations regarding the biology of EGFR mutations and EGFR inhibitors. They confirm that EGFR is the bona fide target of gefitinib in these cell lines, as introduction of a mutant EGFR conferred resistance to gefitinib. Interestingly, WT/T790M was able to exert partial resistance to gefitinib in $\mathrm{H} 3255$ cells (which naturally contains a L858R mutation), while HCC827 cells (which naturally harbor an exon 19 deletion) remained sensitive to gefitinib (Figure 3, A and B). Recent clinical studies have also demonstrated that EGFR inhibitors are more effective in patients with exon 19 deletion mutations than in those with L858R mutations $(49,50)$. Patients with exon 19 deletion mutations have significantly slower disease progression and longer overall survival when treated with gefitinib or erlotinib compared with those with L858R mutations. Accordingly, though both $\mathrm{H} 3255$ and HCC 827 are gefitinib-sensitive cell lines, the HCC827 cells are substantially more sensitive to gefitinib and CL-387,785 (Table 1). Thus it is tempting to speculate that L858R-containing lung cancer cells and tumors are relatively more resistant to gefitinib and that introduction of WT/T790M is a sufficient boost to render them partially resistant to gefitinib. In contrast, the modest amount of resistance promoted by a WT/ T790M allele was inconsequential in the highly sensitive exon 19 deletion cancer cells.

Finally, our studies also provide models in which to test future EGFR inhibitors. Treatment of H3255 Del/T790M, H3255 L858R/T790M, and H3255 GR with CL-387,785 did not restore their sensitivity to that observed with the parental cell line (compare Figure 2C with Figure 3, A and E). Identification of an EGFR inhibitor that is more effective than CL-387,785 in vitro would aid in the prioritization of such an agent for clinical trials for patients who develop T790M-mediated resistance to gefitinib.

\section{Methods}

Cell culture and reagents. The EGFR mutant NSCLC cell lines H3255, HCC827, and H1975 (which naturally harbor L858R, del E746_A750, and L858R/T790M mutations, respectively) were used in this study and have been extensively characterized previously $(8,15,16,22,30,51)$. H3255 and HCC827 are heterozygous for an EGFR mutation and are amplified at the EGFR locus $(15,16)$. Gefitinib was a gift of AstraZeneca. Stock solutions of $10 \mathrm{mM}$ gefitinib were prepared in DMSO and stored at $-20^{\circ} \mathrm{C}$. CL-387,785 was purchased from Calbiochem.

Anti-p-Akt (Ser-473), anti-total Akt, anti-EGFR, and anti-p-ErbB-3 (Tyr-1289) antibodies were obtained from Cell Signaling Technology. Anti-ErbB-3 antibody was obtained from Lab Vision Corp. The p-EGFR (pY1068), total ERK1/2, and p-ERK1/2 (pT185/pY187) antibodies were purchased from BioSource International Inc. Anti-p85 antibody was obtained from Upstate USA Inc.

Cell proliferation and growth assays. Growth and inhibition of growth were assessed by MTS assay. This assay, a colorimetric method for determining the number of viable cells based on the bioreduction of MTS by cells to a formazan product that is soluble in cell culture medium and can be detected spectrophotometrically, was performed according to previously established methods $(8,16,30)$. The cells were exposed to treatment for 72 hours, and the number of cells used per experiment was determined empirically and has been as previously established $(16,30)$. All experimental points were set up in 6-12 wells. Each concentration of drug was performed 6 times, and the mean and SD are shown. Independent experiments were performed at least 3 times, and representative results are shown. The data were graphically displayed using GraphPad Prism version 3.00 for Windows (GraphPad Software). The curves were fitted using a nonlinear regression model with a sigmoidal dose response.

Immunoblotting. Cells grown under the previously specified conditions were lysed in the following lysis buffer: $20 \mathrm{mM}$ Tris, pH 7.4; $150 \mathrm{mM} \mathrm{NaCl}$; 1\% Nonidet P-40; 10\% glycerol; 1 mM EDTA; 1 mM EGTA; 5 mM sodium pyrophosphate; $50 \mathrm{mM} \mathrm{NaF} ; 10 \mathrm{nM} \beta$-glycerophosphate; $1 \mathrm{mM}$ sodium vanadate; $0.5 \mathrm{mM}$ DTT; $4 \mu \mathrm{g} / \mathrm{ml}$ leupeptin; $4 \mu \mathrm{g} / \mathrm{ml}$ pepstatin; $4 \mu \mathrm{g} / \mathrm{ml}$ apoprotein; and $1 \mathrm{mM} \mathrm{PMSF}$. After cell lysis, lysates were centrifuged at $16,000 \mathrm{~g}$ for 5 minutes at $4^{\circ} \mathrm{C}$. The supernatant was used for subsequent procedures. Western blot analyses were conducted after separation by SDS/PAGE electrophoresis and transfer to nitrocellulose membranes. Immunoblotting was performed according to the antibody manufacturers' recommendations. Antibody binding was detected using an enhanced chemiluminescence system (New England Nuclear Life Science Products Inc.). Bands were quantified using NIH ImageJ version 1.36b software (http://rsb.info.nih.gov/ij/). Multiple film exposures were used for quantification so that comparisons were made between lanes within the linear range of the film.

Generation of in vitro H3255 GR. In order to generate a resistant cell line, $\mathrm{H} 3255$ cells were exposed to increasing concentrations of gefitinib in a manner similar to previously described methods using imatinib (28). The cells 
were initially treated with $40 \mathrm{nM}$ gefitinib, equivalent to $\mathrm{IC}_{30}$ in parental $\mathrm{H} 3255$ (Figure 1A). The gefitinib concentrations was increased stepwise to $55 \mathrm{nM}, 75 \mathrm{nM}$, and $100 \mathrm{nM}$, equivalent to $\mathrm{IC}_{40}, \mathrm{IC}_{50}$, and $\mathrm{IC}_{60}$ in parental cells, respectively, when the cells resumed near-normal growth kinetics. The cells that were able to grow in $100 \mathrm{nM}$ gefitinib were obtained 6 months after initial drug exposure. To confirm the emergence of a resistant clone, MTS assays were performed following growth at each concentration after allowing the cells to grow in drug-free condition for at least 4 days. H3255 cells were maintained concomitantly without gefitinib, and their sensitivity to gefitinib was examined every 5 passages. There was no significant change in the sensitivity to gefitinib in parental cells during the period (data not shown).

SURVEYOR analyses. EGFR exon 20 was amplified using previously described primers and conditions (34). Following PCR amplification, the product was digested with SURVEYOR according the manufacturer's recommended conditions (Transgenomic). The digested products were separated by HPLC column using the WAVE 3500HT High Sensitivity System (Transgenomic) using previously described methods (34). In order to determine whether T790M was cis to L858R in H3255 GR, RT-PCR products were cloned into pCR Blunt (Invitrogen), and pools of 8 individual clones were screened using SURVEYOR followed by direct sequencing. SURVEYOR analyses also confirmed the L858R mutations in H3255 and H1975 DNA (data not shown).

PCR analyses of H3255 GR cells. H3255 GR cells were counted by Coulter counter and seeded in PBS at 20 cells/well in 96-well plates. The number of cells per well was confirmed by counting the number of cells per well in a replica plate seeded in growth media. One-tenth the volume ( 2 cells) was spiked into a 50- $\mu$ l PCR reaction via JumpStart (Sigma-Aldrich) using primers to amplify exon 20. One microliter of the initial reaction was used for a subsequent PCR reaction with nested primers. A portion of the PCR was run on a $2 \%$ agarose gel to confirm the presence of a single band of the correct molecular weight. Samples that produced a PCR product were submitted to SURVEYOR for analysis. Negative controls with no template added were employed at all PCR steps. Oligonucleotide sequences are shown in Supplemental Methods.

EGFR sequencing. RNA was isolated from cell lines using the RNEasy RNA isolation kit (Qiagen). cDNA was transcribed from $2 \mu \mathrm{g}$ total RNA with Superscript II Reverse Transcriptase (Invitrogen). The cDNA was used as a template for subsequent PCR amplifications of EGFR. The entire EGFR coding region was sequenced. The details of the PCR conditions and the primers have been previously published (16). NSCLC patients' tumor specimens were sequenced in the CLIA-certified Harvard Laboratory for Molecular Medicine using previously described methods (8). All patients provided informed consent for EGFR sequencing, and the studies were approved by the Institutional Review Board of the Dana-Farber Cancer Institute.

EGFR mutant constructs and retroviral infection. The human EGFR cDNA coding region (GenBank accession no. X00588) was cloned into pDNRDual (BD Biosciences). The EGFR mutants (L858R and L747_S752del, P753S) were constructed with Quick Change Site-Directed Mutagenesis kit (Stratagene) according to the manufacturer's instructions and as previously described (30). The T790M mutation was introduced into EGFR WT or L858R or L747_S752del, P753S constructs using site-directed mutagenesis. Oligonucleotide sequences are shown in Supplemental Methods. All constructs were confirmed by DNA sequencing. As a control a retroviral construct expressing GFP was used that has been previously described (26). The ca-Akt construct (myristoylated Akt), a kind gift from T. Obata (University of Tokushima, Tokushima, Japan), was also cloned into pDNR-Dual (BD Biosciences) (52). The HA-tagged $\mathrm{p} 110 \alpha$ E545K construct has been previously described (37). All constructs (including GFP) were shuttled into the retroviral vector JP1520 using the BD Creator System (BD Biosciences). NSCLC cells were infected with retrovirus according to standard protocols as described previously $(26,53)$. Stable populations were obtained by selection in media containing $2 \mu \mathrm{g} / \mathrm{ml}$ puromycin (Sigma-Aldrich). EGFR expression levels in the transfected cells were analyzed by quantitative realtime PCR using previously established methods (54).

EGFR shRNA constructs and lentiviral infection. EGFR shRNA constructs cloned in pLKO.1 puro vector were obtained from Harvard RNAi consortium. Each construct contained a 21-bp sequence targeting different regions of EGFR, a 6-nuecleotide hairpin sequence (CTCGAG), and a 21-bp complementary strand sequence. shRNA constructs $B$ and $C$ targeted the $3^{\prime}$ untranslated region of EGFR, while constructs A, D, and E targeted the coding region. The specific shRNA sequences are shown in Supplemental Methods. Lentivirus production and infections were performed as previously described (26). MTS assays were performed 7 days after infection. Results were unchanged when performed under puromycin selection.

Xenograft studies. Nude mice ( $(n u / n u ; 6-8$ weeks old; Charles River Laboratories) were used for in vivo studies and were cared for in accordance with the standards of the Institutional Animal Care and Use Committee (IACUC) under a protocol approved by the Animal Care and Use Committee of Children's Hospital Boston. Mice were anesthetized using a 2\% Isoflurane (Baxter) inhalation oxygen mixture. A suspension of $5 \times 10^{6}$ HCC827 GFP or HCC827 Del/T790M lung cancer cells (in $0.2 \mathrm{ml} \mathrm{PBS}$ ) were inoculated subcutaneously into the lower-right quadrant of the flank of each mouse. Five mice were inoculated with either HCC827 GFP or HCC827 Del/T790M cells in the gefitinib treatment group. Tumors were measured twice weekly using calipers, and volume was calculated as length $\times$ width $^{2} \times 0.52$. Mice were monitored daily for body weight and general condition. Mice were randomized to treatment when the mean tumor volume was $400-500 \mathrm{~mm}^{3}$. Gefitinib was administered at $150 \mathrm{mg} / \mathrm{kg} / \mathrm{d}$ by daily oral gavage. This dose schedule was previously reported to be highly efficacious in other tumor models, and doses were at or below the respective maximum well-tolerated doses in nude mice (55). The experiment was terminated when the mean size of the control tumors reached $2,000 \mathrm{~mm}^{3}$.

PI3K assay. The PI3K assays were performed according to previously described methods (37). ErbB-3 was immunoprecipitated overnight at $4{ }^{\circ} \mathrm{C}$ from either control or gefitinib-treated NSCLC cell lines. The immunoprecipitate was captured on protein G-Sepharose beads. Half of the immunoprecipitate was used for Western blot analysis, and the other half was used for PI3K assays; each was performed in triplicate. The relative amounts of ErbB-3 in the immunoprecipitates were determined by Western blot analyses using the LI-COR Odyssey protein quantification system. The average and standard deviation of the PI3K activity normalized to the amount of ErbB-3 protein is shown in relative units as a percent of control (no gefitinib).

EGFR immunofluorescence. The p-EGFR (pY1068) antibody (Cell Signaling Technology) was used for these studies according to the manufacturer's recommendations. Cells were plated on coverslips and, following gefitinib and/or EGF treatment (100 ng/ml), fixed using 4\% paraformaldehyde. The coverslips were washed, blocked in blocking buffer $(5 \%$ goat serum, $1 \%$ BSA, $0.02 \% \mathrm{NaN}_{3}$, and $1 \times \mathrm{PBS}$ ) for 45 minutes at room temperature, and stained with the p-EGFR antibody overnight at $4^{\circ} \mathrm{C}$ according to the manufacturer's recommended conditions. Alexa Fluor 594 goat anti-mouse secondary antibody (Invitrogen) was used at a 1:500 dilution. Images were obtained on a NikoTE200 with a PerkinElmer Spinning Disk Confocal Microscope.

Statistics. In order to compare effects of gefitinib in the different xenografts, the data were transformed into the log scale for analysis in order to approximate a linear fit, reflecting the exponential growth of tumors. Some tumors of the gefitinib-treated mice in the HCC827 GFP model had shrunk to the extent that they were no longer measurable. Their tumor volumes were recoded arbitrarily as $0.01 \mathrm{~mm}^{3}$, that is, 2 logs lower than the smallest nonzero tumor volume. As the animals were randomized among treatment groups, an overall mean was assumed for the population of mouse-specific intercepts in each xenograft model. Linear and quadratic terms were fitted as regression effects of treatment in order to model the 
initial dip in tumor growth observed in the gefitinib-treated group of the HCC827 Del/T790M model. Growth patterns between groups were compared by the appropriate contrast of fixed regression effects using inference based on the $F$ test. Estimation by restricted maximum likelihood was computed using SAS (version 9.1; SAS Institute Inc.), and model fit was assessed by Akaike information criterion.

The paired Student's $t$ test was used to determine whether growth following shRNA infection of construct B was different compared with that following infection of control construct A. Statistical analyses were performed using StatView (version 5.01; SAS Institute Inc.). All statistical tests were 2 -sided. A $P$ value less than 0.05 was considered to be statistically significant.

\section{Acknowledgments}

This study was supported by NIH grants 1K12CA87723-01 (to Pasi A. Jänne), 1RO1CA114465-01 (to Bruce E. Johnson and Pasi A. Jänne), and GM41890 and PO1 CA089021 (to Lewis C. Cantley); National Cancer Institute grant K08CA120060-01 (to Jeffrey A. Engelman); National Cancer Institute Lung SPORE grant P20CA90578-02 (to Bruce E. Johnson and Beow Y. Yeap); the American Society of Clinical Oncology (Jeffrey A. Engelman); the American Association for Cancer Research (Jeffrey A. Engelman); and the International Association for the Study of Lung Cancer (Jeffrey A. Engelman). The authors would like to thank Ed Fox for EGFR sequencing, Karen Cichowski for critical review of this manuscript and helpful discussion, and the laboratories of Jeffrey Settleman and Daniel Haber for their helpful discussions and assistance with lentiviral experiments.

Received for publication March 27, 2006, and accepted in revised form July 18, 2006.

Address correspondence to: Lewis C. Cantley, Harvard Institutes of Medicine, 77 Avenue Louis Pasteur, Room 1022, Boston, Massachusetts 02115, USA. Phone: (617) 667-0934; Fax: (617) 667-0957; E-mail: lewis_cantley@hms.harvard.edu.

Jeffrey A. Engelman and Toru Mukohara contributed equally to this work.
1. Rusch, V., et al. 1997. Overexpression of the epidermal growth factor receptor and its ligand transforming growth factor alpha is frequent in resectable non-small cell lung cancer but does not predict tumor progression. Clin. Cancer Res. 3:515-522.

2. Fontanini, G., et al. 1998. Evaluation of epidermal growth factor-related growth factors and receptors and of neoangiogenesis in completely resected stage I-IIIA non-small-cell lung cancer: amphiregulin and microvessel count are independent prognostic indicators of survival. Clin. Cancer Res. 4:241-249.

3. Brabender, J., et al. 2001. Epidermal growth factor receptor and HER2-neu mRNA expression in nonsmall cell lung cancer is correlated with survival. Clin. Cancer Res. 7:1850-1855.

4. Shepherd, F.A., et al. 2005. Erlotinib in previously treated non-small-cell lung cancer. N. Engl. J. Med. 353:123-132.

5. Fukuoka, M., et al. 2003. Multi-institutional randomized phase II trial of gefitinib for previously treated patients with advanced non-small-cell lung cancer. J. Clin. Oncol. 21:2237-2246.

6. Kris, M.G., et al. 2003. Efficacy of gefitinib, an inhibitor of the epidermal growth factor receptor tyrosine kinase, in symptomatic patients with nonsmall cell lung cancer: a randomized trial. JAMA. 290:2149-2158.

7. Perez-Soler, R., et al. 2004. Determinants of tumor response and survival with erlotinib in patients with non-small-cell lung cancer. J. Clin. Oncol. 22:3238-3247.

8. Paez, J.G., et al. 2004. EGFR mutations in lung cancer: correlation with clinical response to gefitinib therapy. Science. 304:1497-1500.

9. Lynch, T.J., et al. 2004. Activating mutations in the epidermal growth factor receptor underlying responsiveness of non-small-cell lung cancer to gefitinib. N. Engl.J. Med. 350:2129-2139.

10. Pao, W., et al. 2004. EGF receptor gene mutations are common in lung cancers from "never smokers" and are associated with sensitivity of tumors to gefitinib and erlotinib. Proc. Natl. Acad. Sci. U. S. A. 101:13306-13311.

11. Mitsudomi, T., et al. 2005. Mutations of the epidermal growth factor receptor gene predict prolonged survival after gefitinib treatment in patients with non-small-cell lung cancer with postoperative recurrence. J. Clin. Oncol. 23:2513-2520.

12. Han, S.W., et al. 2005. Predictive and prognostic impact of epidermal growth factor receptor mutation in non-small-cell lung cancer patients treated with gefitinib. J. Clin. Oncol. 23:2493-2501.

13. Takano, T., et al. 2005. Epidermal growth factor receptor gene mutations and increased copy numbers predict gefitinib sensitivity in patients with recurrent non-small-cell lung cancer. J. Clin. Oncol. 23:6829-6837.

14. Cappuzzo, F., et al. 2005. Epidermal growth factor receptor gene and protein and gefitinib sensitivity in non-small-cell lung cancer. J. Natl. Cancer Inst. 97:643-655.

15. Amann, J., et al. 2005. Aberrant epidermal growth factor receptor signaling and enhanced sensitivity to EGFR inhibitors in lung cancer. Cancer Res. 65:226-235.

16. Tracy, S., et al. 2004. Gefitinib induces apoptosis in the EGFRL858R non-small-cell lung cancer cell line H3255. Cancer Res. 64:7241-7244.

17. Bianco, R., et al. 2003. Loss of PTEN/MMAC1/TEP in EGF receptor-expressing tumor cells counteracts the antitumor action of EGFR tyrosine kinase inhibitors. Oncogene. 22:2812-2822.

18. She, Q.B., et al. 2005. The BAD protein integrates survival signaling by EGFR/MAPK and PI3K/Akt kinase pathways in PTEN-deficient tumor cells. Cancer Cell. 8:287-297.

19. Baselga, J., et al. 2005. Phase II and tumor pharmacodynamic study of gefitinib in patients with advanced breast cancer. J. Clin. Oncol. 23:5323-5333.

20. Gorre, M.E., Ellwood-Yen, K., Chiosis, G., Rosen, N., and Sawyers, C.L. 2002. BCR-ABL point mutants isolated from patients with imatinib mesylateresistant chronic myeloid leukemia remain sensitive to inhibitors of the BCR-ABL chaperone heat shock protein 90. Blood. 100:3041-3044.

21. Antonescu, C.R., et al. 2005. Acquired resistance to imatinib in gastrointestinal stromal tumor occurs through secondary gene mutation. Clin. Cancer Res. 11:4182-4190.

22. Pao, W., et al. 2005. Acquired resistance of lung adenocarcinomas to gefitinib or erlotinib is associated with a second mutation in the EGFR kinase domain. PLoS Med. 2:e73.

23. Kwak, E.L., et al. 2005. Irreversible inhibitors of the EGF receptor may circumvent acquired resistance to gefitinib. Proc. Natl. Acad. Sci. U. S. A. 102:7665-7670.

24. Kobayashi, S., et al. 2005. EGFR mutation and resistance of non-small-cell lung cancer to gefitinib. N. Engl. J. Med. 352:786-792.

25. Kobayashi, S., et al. 2005. An alternative inhibitor overcomes resistance caused by a mutation of the epidermal growth factor receptor. Cancer Res. 65:7096-7101.
26. Engelman, J.A., et al. 2005. ErbB-3 mediates phosphoinositide 3-kinase activity in gefitinib-sensitive non-small cell lung cancer cell lines. Proc. Natl. Acad. Sci. U. S. A. 102:3788-3793.

27. Fujimoto, N., et al. 2005. High expression of ErbB family members and their ligands in lung adenocarcinomas that are sensitive to inhibition of epidermal growth factor receptor. Cancer Res. 65:11478-11485.

28. Ricci, C., et al. 2002. Mutation in the ATP-binding pocket of the ABL kinase domain in an STI571resistant BCR/ABL-positive cell line. Cancer Res. 62:5995-5998.

29. Albanell, J., et al. 2002. Pharmacodynamic studies of the epidermal growth factor receptor inhibitor ZD1839 in skin from cancer patients: histopathologic and molecular consequences of receptor inhibition. J. Clin. Oncol. 20:110-124.

30. Mukohara, T., et al. 2005. Differential effects of gefitinib and cetuximab on non-small-cell lung cancers bearing epidermal growth factor receptor mutations. J. Natl. Cancer Inst. 97:1185-1194.

31. Janmaat, M.L., Kruyt, F.A., Rodriguez, J.A., and Giaccone, G. 2003. Response to epidermal growth factor receptor inhibitors in non-small cell lung cancer cells: limited antiproliferative effects and absence of apoptosis associated with persistent activity of extracellular signal-regulated kinase or Akt kinase pathways. Clin. Cancer Res. 9:2316-2326.

32. Brehmer, D., et al. 2005. Cellular targets of gefitinib. Cancer Res. 65:379-382.

33. Fabian, M.A., et al. 2005. A small molecule-kinase interaction map for clinical kinase inhibitors. Nat. Biotechnol. 23:329-336.

34. Janne, P.A., et al. 2006. A rapid and sensitive enzymatic method for epidermal growth factor receptor mutation screening. Clin. Cancer Res. 12:751-758.

35. Samuels, Y., et al. 2004. High frequency of mutations of the PIK3CA gene in human cancers. Science. 304:554.

36. Samuels, Y., et al. 2005. Mutant PIK3CA promotes cell growth and invasion of human cancer cells. Cancer Cell. 7:561-573.

37. Isakoff, S.J., et al. 2005. Breast cancer-associated PIK3CA mutations are oncogenic in mammary epithelial cells. Cancer Res. 65:10992-11000.

38. Kang, S., Bader, A.G., and Vogt, P.K. 2005. Phosphatidylinositol 3-kinase mutations identified in human cancer are oncogenic. Proc. Natl. Acad. Sci. U. S. A. 102:802-807.

39. Greulich, H., et al. 2005. Oncogenic transformation by inhibitor-sensitive and -resistant EGFR 
mutants. PLoS Med. 2:e313.

40. Bell, D.W., et al. 2005. Inherited susceptibility to lung cancer may be associated with the T790M drug resistance mutation in EGFR. Nat. Genet. 37:1315-1316.

41. Toyooka, S., Kiura, K., and Mitsudomi, T. 2005. EGFR mutation and response of lung cancer to gefitinib. N. Engl. J. Med. 352:2136; author reply 2136.

42. Tabone, S., et al. 2005. KIT overexpression and amplification in gastrointestinal stromal tumors (GISTs). Biochim. Biophys. Acta. 1741:165-172.

43. Debiec-Rychter, M., et al. 2005. Mechanisms of resistance to imatinib mesylate in gastrointestinal stromal tumors and activity of the PKC412 inhibitor against imatinib-resistant mutants. Gastroenterology. 128:270-279.

44. Hochhaus, A., et al. 2002. Molecular and chromosomal mechanisms of resistance to imatinib (STI571) therapy. Leukemia. 16:2190-2196.

45. Mellinghoff, I.K., et al. 2005. Molecular determinants of the response of glioblastomas to EGFR kinase inhibitors. N. Engl. J. Med. 353:2012-2024. 46. Slamon, D.J., et al. 1989. Studies of the HER-2/neu proto-oncogene in human breast and ovarian cancer. Science. 244:707-712.

47. Levine, R.L., et al. 2005. Activating mutation in the tyrosine kinase JAK2 in polycythemia vera, essential thrombocythemia, and myeloid metaplasia with myelofibrosis. Cancer Cell. 7:387-397.

48. Nagata, Y., et al. 2004. PTEN activation contributes to tumor inhibition by trastuzumab, and loss of PTEN predicts trastuzumab resistance in patients. Cancer Cell. 6:117-127.

49. Jackman, D.M., et al. 2006. Exon 19 deletion mutations of epidermal growth factor receptor are associated with prolonged survival in non-small cell lung cancer patients treated with gefitinib or erlotinib. Clin. Cancer Res. 12:3908-3914.

50. Riely, G.J., et al. 2006. Clinical course of patients with non-small cell lung cancer and epidermal growth factor receptor exon 19 and exon 21 mutations treated with gefitinib or erlotinib. Clin. Cancer
Res. 12:839-844.

51. Fujishita, T., et al. 2003. Sensitivity of non-smallcell lung cancer cell lines established from patients treated with prolonged infusions of Paclitaxel. Oncology. 64:399-406.

52. Namikawa, K., et al. 2000. Akt/protein kinase B prevents injury-induced motoneuron death and accelerates axonal regeneration. J. Neurosci. 20:2875-2886.

53. Zhao, J.J., et al. 2003. Human mammary epithelial cell transformation through the activation of phosphatidylinositol 3-kinase. Cancer Cell. 3:483-495.

54. Zhao, X., et al. 2005. Homozygous deletions and chromosome amplifications in human lung carcinomas revealed by single nucleotide polymorphism array analysis. Cancer Res. 65:5561-5570.

55. Sirotnak, F.M., Zakowski, M.F., Miller, V.A., Scher, H.I., and Kris, M.G. 2000. Efficacy of cytotoxic agents against human tumor xenografts is markedly enhanced by coadministration of ZD1839 (Iressa), an inhibitor of EGFR tyrosine kinase. Clin. Cancer Res. 6:4885-4892. 